\title{
Los asesores de tesis: ¿cumplen criterios de autoría?
}

\author{
Thesis advisers: do they meet criteria for author- \\ ship?
}

\author{
Orientadores de tese: eles atendem aos critérios de \\ autoria?
}

\author{
Cristian Díaz-Vélez 1,2 \\ Jorge Fernández-Mogollón 2 \\ Moisés Apolaya-Segura 1 \\ Yhojar Pisfil-Farroñay ${ }^{3}$
}

doi: 10.1590/0102-311X00251318
Estimadas Editoras,

En el Editorial mencionan los criterios de autoría para los artículos publicados en la revista 1, pero nos preguntamos si esos criterios son cumplidos por los asesores de tesis.

En el Perú, según la Superintendencia Nacional de Educación Superior Universitaria (SUNEDU) es un requisito mínimo el realizar una investigación científica para obtener el grado académico de bachiller, maestría, doctorado, título y segunda especialidad profesional 2. Éste pone a prueba la habilidad y capacidad adquirida de los estudiantes de pregrado y postgrado en el área de investigación 3.

Para llevarlo a cabo también es necesaria la participación del asesor, ya que por su experiencia en el campo de la investigación debe orientar al estudiante en la elección del tema, análisis, redacción, revisión crítica y posteriormente su publicación 4 . Cabe resaltar que pueden existir dos tipos de asesores en las investigaciones científicas: asesor metodológico y asesor temático o especialista en el campo que se desarrollará el estudio 5 .

En un estudio realizado en un grupo de egresados de diferentes universidades por Tresierra-Ayala et al. 5 se evidenció en cerca de la mitad de los estudios que el asesoramiento fue mayor en el campo metodológico que en el campo temático, y la otra mitad manifestó que tenían casi nada o nada de asesoría; por lo que es posible que muchos de estos asesores no cumplan con los criterios de autoría. Los criterios de autoría son dados por el Comité Internacional de Editores de Revistas Médicas (ICMJE) 6, los cuales son: (1) contribuciones significativas a la concepción o diseño del manuscrito, o a la recolección, análisis o interpretación de los datos; (2) redacción o revisión crítica importante del contenido del manuscrito; (3) la aprobación final de la versión que se publicará; y (4) la asunción de la responsabilidad frente a todos los aspectos del manuscrito, para garantizar que los asuntos relativos a la exactitud o integridad de cualquier parte del mismo serán adecuadamente investigados y resueltos.

Considerando lo antes mencionado, se aplicó un cuestionario anónimo a 59 estudiantes de medicina que habían concluido una investigación científica para la obtención de un título profesional en universidades de Lambayeque (Perú), quienes presentaban un asesor responsable del seguimiento metodológico (asesor metodológico) y otro asesor responsable de la parte temática (asesor temático) de la investigación.

Se encontró que el mayor porcentaje de cumplimiento de criterios de autoría se encuentra con los asesores metodológicos (Tabla 1), aunque este resultado podría estar influenciado por la función
${ }_{1}^{1}$ Facultad de Medicina Universidad de San Martín de Porres, Chiclayo, Perú. 2 Hospital Nacional Almanzor Aguinaga Asenjo, Lambayeque, Perú.

3 Facultad de Medicina, Universidad Señor de Sipán, Chiclayo, Perú.

\section{Correspondencia} C. Díaz-Vélez Res. Villa Bouganville A4, Reque / Chiclayo - 05174, Perú.

cristiandiazv@hotmail.com 
Tabla 1

Percepción del cumplimiento de criterios de autoría de asesores de investigaciones para obtener un título profesional estudiantes de medicina.

\begin{tabular}{lccccc}
\hline Criterios de autoría & \multicolumn{2}{c}{ Asesor metodológico } & \multicolumn{2}{c}{ Asesor temático } & \multicolumn{2}{c}{ Valor de $\mathbf{p}$} \\
& $\mathbf{n}$ & $\mathbf{\%}$ & $\mathbf{n}$ & $\mathbf{\%}$ & \\
\hline Concepción de la idea & 31 & 52,5 & 29 & 49,2 & 0,85 \\
Diseño del manuscrito & 43 & 72,9 & 20 & 33,9 & $<0,001$ \\
Recolección de datos & 15 & 25,4 & 23 & 39,0 & 0,16 \\
Análisis de datos & 35 & 59,3 & 16 & 27,1 & $<0,001$ \\
Interpretación de datos & 40 & 67,8 & 18 & 30,5 & $<0,001$ \\
Redacción del manuscrito & 37 & 62,7 & 17 & 28,8 & $<0,001$ \\
Revisión crítica del manuscrito & 48 & 81,4 & 39 & 66,1 & 0,09 \\
Cumple criterios de autoría & 37 & 62,7 & 15 & 25,4 & $<0,001$ \\
\hline
\end{tabular}

de realizar el seguimiento continuo asignado durante el curso curricular.

No cumplir con los criterios de autoría por parte de los asesores de un trabajo de investigación, también se consideraría una falta ética que daña la integridad del sistema de autoría de las publicaciones científicas. Así como también la incorporación de los autores "honorarios", que son personas que no aportaron científicamente, pero tienen gran prestigio y de esa forma aumentan la credibilidad de las publicaciones. No obstante, existen personas que son contratadas para redactar y aportar a los trabajos de investigación, pero están desvinculados del proyecto, los llamados autores "fantasma"; generalmente esto se ve en las industrias farmacéuticas 7,8 .

Las controversias relacionadas con las autorías cada vez son mayores, por eso es necesario que los autores y co-autores cumplan los requisitos de las mismas; y de esa forma asumir la responsabilidad que esta conlleva, dejar un aporte científico y no sólo hacerlo por aumentar nuestros méritos profesionales o sólo por justificar el curriculum vitae 8 . 


\section{Colaboradores}

C. Díaz-Vélez formuló la idea del manuscrito. Todos los autores contribuyeron con el diseño, redacción y revisión crítica.

\section{Informaciones adicionales}

ORCID: Cristian Díaz-Vélez (0000-0003-45932509); Jorge Fernández-Mogollón (0000-00018293-0882); Moisés Apolaya-Segura (0000-00015650-9998); Yhojar Pisfil-Farroñay (0000-00028863-0327).

\section{Referencias}

1. Pietrukowicz MCLC, Carvalho L, Ribeiro CK. La autoría científica en CSP. Cad Saúde Pública 2018; 34:e00174218.

2. Superintendencia Nacional de Educación $\mathrm{Su}-$ perior Universitaria. Reglamento del registro nacional de grados y títulos. https://www.su nedu.gob.pe/reglamentodelregistronacional degradosytitulos/ (accedido el 15/Jul/2018).

3. Atamuri-Anahui N. Publicación científica de asesores de tesis de pregrado en una escuela de medicina de Cusco, Perú. Investigación en Educación Médica 2016; 5:279-80.

4. Oyola-Garcia A. El asesor de tesis. Acta Méd Peru 2015; 32:131-2.

5. Tresierra-Ayala M, Pinco-Cabanillas C, DíazVélez C. Percepción del asesoramiento y defensa del trabajo de grado por egresados de medicina de universidades del norte del Perú. Revista Médica de Trujillo 2017; 12:95-8.

6. International Committee of Medical Journal Editors. Defining the role of authors and contributors. http://www.icmje.org/recommen dations/browse/roles-and-responsibilities/ defining-the-role-of-authors-and-contribu tors.html (accedido el 24/Jul/2018).

7. Sarasa N. Los tutores de tesis en los procesos académicos del postgrado en las ciencias médicas. Edumecentro 2014; 6:1-5.

8. Gisbert J, Piqué J. Hablemos de autoría de las publicaciones científicas. GH Continuada 2009; 8:53-61.

Recibido el 29/Dic/2018

Aprovado el 11/Ene/2019 